\title{
Estudo da hidrodinâmica de diferentes geometrias de sítios de conversão de energia das correntes
}

\author{
Studying the conversion energy on different geometry configuration of current power turbines \\ site \\ B. C. Pezzato ${ }^{1 *}$, E. P. Kirinus ${ }^{1}$, W. C. Marques ${ }^{2}$ \\ ${ }^{1}$ Instituto de oceanografia. Universidade Federal do Rio Grande, Rio Grande-RS, Brasil \\ ${ }^{2}$ Institudo de matemática, estatistica e física. Universidade Federal do Rio Grande, Rio Grande-RS, Brasil \\ *bruno_pezzato@hotmail.com
}

(Recebido em 19 de setembro de 2014; aceito em 20 de outubro de 2014)

\begin{abstract}
O crescente aumento da população mundial acarreta em um consumo de energia elétrica cada vez maior, sendo a maior parte da energia do mundo gerada a partir de combustíveis fosseis, intensificando a busca pelo desenvolvimento de tecnologias de geração de energia por fontes alternativas. A energia das correntes marinhas é uma fonte alternativa bastante viável, mas que ainda apresenta um alto custo de implementação. Com o desenvolvimento tecnológico este custo tenderá a baixar, então devemos desde já realizar pesquisar para definir os melhores locais de instalação. Na Plataforma Continental Sul Brasileira, existem duas regiões propicias, sendo uma mais adequada. A partir da modelagem numérica utilizando o modelo hidrodinâmico, TELEMAC, com um modulo de conversão de energia, podemos determinar qual seria a melhor geometria para os sítios de turbinas de conversão de energia. Este estudo demonstrou que um sítio com 10 turbinas estando a $45^{\circ}$ em relação à costa apresenta o melhor rendimento.
\end{abstract}

Palavras-chave: Energias renovaveis, correntes costeiras, sitios de conversão

The growth in the world's population implies in a growth of electric energy consumption, being the biggest amount of this energy produced from fossil fuels. In this sense, the technology development of alternative energy production has grown. Ocean currents energy is a feasible alternative energy source; however, the production cost is still high. With technology development, this cost would drop; therefore, research on proper harvesting sites is already desirable. Off the Southern Brazilian coast, there are two viable regions for harvesting, with one of the sites better than the other. Using the TELEMAC model, coupled with a energy conversion module, it is possible to define which is the best turbine field geometry to harvest the energy. This work shows that a farm with ten turbines in a 45 degree angle with the coastline presents the best results.

Keywords: Renewable Energies, Coastal Currents, Farm of turbines

\section{INTRODUÇÃO}

Um dos maiores problemas da humanidade na atualidade é falta de energia, ocasionada tanto pelo aumento do consumo quanto pelo aumento da população. O Consumo cresceu cerca de $70 \%$ nas últimas três décadas [1]. A maior parte desta energia é proveniente de combustíveis fosseis, cerca de 80\% pincipalmente nos Estados Unidos segundo Bollmann [1], que além de não serem renováveis contribuem com o efeito estufa pela liberação de $\mathrm{CO}_{2}$ (Dióxido de Carbono). Tendo isso em vista, a busca por energias renováveis e menos poluentes tem crescido, pois podem suprir parte desta demanda, além de apresentarem um menor impacto ambiental [2].

Inicialmente, energias renováveis apresentavam altos custos de implementação, mas isso vem diminuindo com o passar dos anos com o desenvolvimento de novas tecnologias. Um tipo energia que ainda apresenta um alto custo é a proveniente de correntes marinhas, sendo pouco explorada [3]. Como a maior parte da população mundial se encontra no litoral, a energia elétrica proveniente de correntes marítimas deve ser uma alternativa a ser levada em consideração. 
O Brasil tem um consumo maior que a produção e por isso 8,3\% da sua energia é importada [4]. Apesar de ter uma das maiores faixas costeiras do mundo, aproximadamente $8000 \mathrm{~km}, \mathrm{o}$ país ainda não apresenta nenhum estudo sobre potencial energético utilizável para a conversão em energia elétrica através da utilização de turbinas hidrocinéticas. Esta energia poderia suprir uma parte dessa diferença.

O estado do Rio Grande do Sul no ano de 2009 apresentou um consumo de 26,5 TWh com a possibilidade de alcançar um consumo de 34 TWh em 2015 [4], ilustrando a atual situação do estado e do país.

A utilização da energia das correntes marítimas poderia auxiliar a matriz energética brasileira. Em um estudo preliminar, Marques [5], demonstrou que poderia gerar até 5GW/ano usando 6 conversores, com baixo impacto ambiental. Em um estudo mais aprofundado Kirinus [6], demonstrou que existem duas regiões na Plataforma Continental Sul Brasileira (PCSB), localizada entre $28^{\circ} \mathrm{S}$ e $35^{\circ} \mathrm{S}$ (Figura 1) com bom potencial energético, sendo a melhor região localizada no entorno do Farol da Conceição, distante $11 \mathrm{~km}$ da costa com profundidade de $18 \mathrm{~m}$. Nesta região a produção média de energia pode alcançar $40 \mathrm{MWh}$ e ainda valores integrados de $13 \mathrm{GWh} /$ ano.

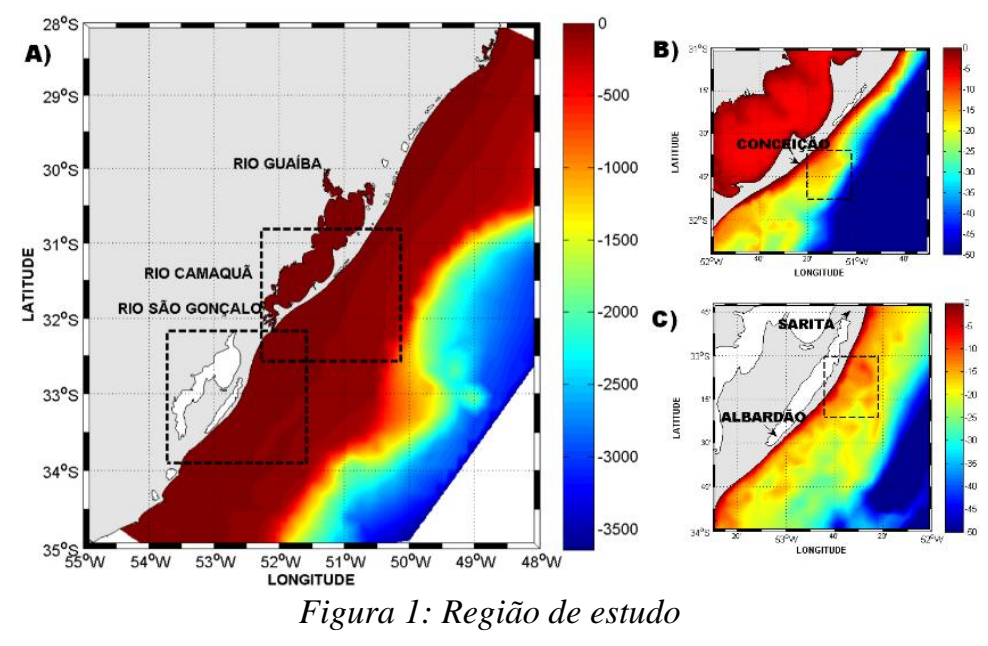

A região da PCSB apresenta uma alta dinâmica devido ao encontro de várias massas de água, tanto de origem tropical, quanto de origem subantártica, além de um grande aporte continental que geram assim fortes gradientes termohalinos. Tem sua circulação influenciada principalmente pelo Rio da Prata, por ventos bidirecionais de Nordeste - Sudoeste e pela corrente de contorno oeste que fluem pela quebra da plataforma [7], além de apresentar uma forte diferença sazonal devido a mudanças do vento. Esse estudo tem por objetivo demonstrar qual seria a melhor orientação das turbinas no sitio próximo ao Farol de Conceição para se alcançar um melhor rendimento na geração de energia elétrica.

\section{MATERIAL E MÉTODOS}

\subsection{Modelo numérico hidrodinâmico}

O modelo numérico para simulações hidrodinâmicas utilizado foi o TELEMAC, (www.opentelemac.org). Esse modelo utiliza as equações de Navier-Strokes considerando às variáveis locais, como a superfície livre do mar, considerando a densidade constante, a pressão hidrostática e as aproximações de Boussinesq. O modelo é baseado em técnicas de elementos finitos para resolver a equação hidrodinâmica [8] e conta com o sistema de coordenadas sigma para discretização vertical. 


\subsection{Modulo de conversão de energia}

O módulo de conversão de energia utiliza a equação padrão de turbinas (equação1) para calcular a energia elétrica gerada, em Watts(W), a partir da velocidade do fluxo da corrente incidente nos conversores. Utilizando o princípio da conservação de energia, a velocidade da corrente é obtida em cada intervalo de tempo através modelo hidrodinâmico que transfere para o módulo de conversão de energia. Uma parte da velocidade é convertida em energia elétrica, a velocidade é então atualizada para manter o balanço de energia do modelo hidrodinâmico, TELEMAC3D.

$$
\mathrm{P}(\mathrm{W})=1 / 2 \rho \mu \mathrm{A} v^{3}
$$

Onde: $\mathrm{P}(\mathrm{W})$ é a energia produzida em Watts, $\rho$ é a densidade do fluido, $\mu$ é o coeficiente de eficiência da turbina, A é a área do conversor e v é a velocidade. A tabela 1 indica os parâmetros utilizados pelo modulo de conversão de energia

Tabela 1: Parâmetros técnicos da turbina.

$\begin{array}{cc}$\cline { 2 - 2 } \text {$\left.Parâmetro } & \text { Valor } \\ \hline \begin{array}{c}\text { Velocidade de início } \\ \text { de movimento }\end{array} & 0,2 \mathrm{~m} / \mathrm{s} \\ \begin{array}{c}\text { Velocidade nominal } \\ \text { Coeficiente de }\end{array} & 1,5 \mathrm{~m} / \mathrm{s} \\ \text { eficiência da turbina } \\ (\mu)\end{array}\right)$

Para o estudo do potencial de conversão de energia e a influência dos conversores sobre os processos hidrodinâmicos da PCSB, foram realizadas quatro simulações de 25 dias aplicando os mesmos parâmetros físicos. O período simulado compreende entre 01 e 25 de janeiro de 2004.

O sítio de conversão apresenta 10 turbinas dispostas de diferentes maneiras (Figura 2), com 500 metros de distância entre cada turbina tanto na direção X quanto Y. Por conta de limitações computacionais a turbina é caracterizada por 4 pontos com 10 metros de distância entre cada um (Figura 3a).

O modelo de conversão interage com a turbina, adquirindo velocidade da corrente nos pontos vermelhos (Figura 3a), que é convertida em energia. A perda de energia cinética é liberada nos pontos amarelos (Fig. 3a). As turbinas são consideradas como colunas no fundo oceânico (Figura 3b), com o intuito de simular a estrutura das turbinas.

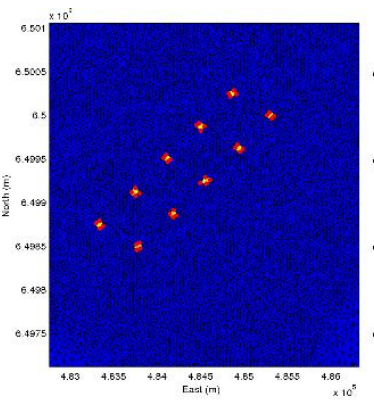

(a)

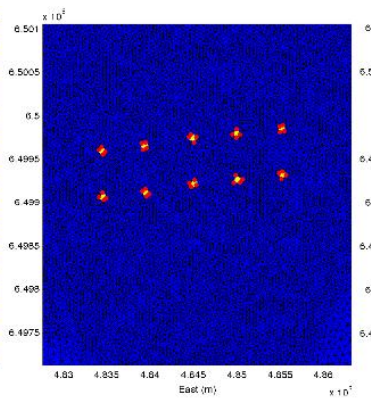

(b)

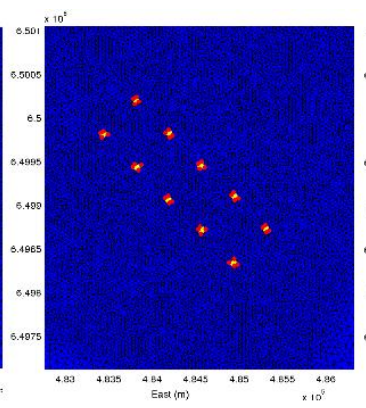

(c)

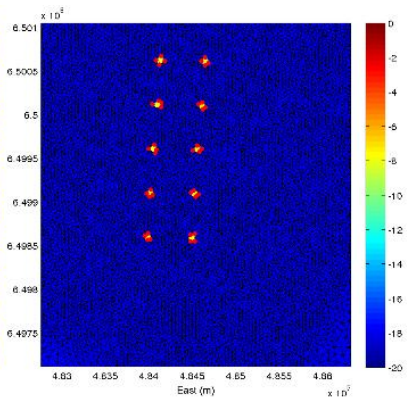

(d)

Figura 2: Disposição dos conversores nos diferentes cenários. (a) Cenário I. (b) Cenário II. (c) Cenário III. (d) Cenário IV. 


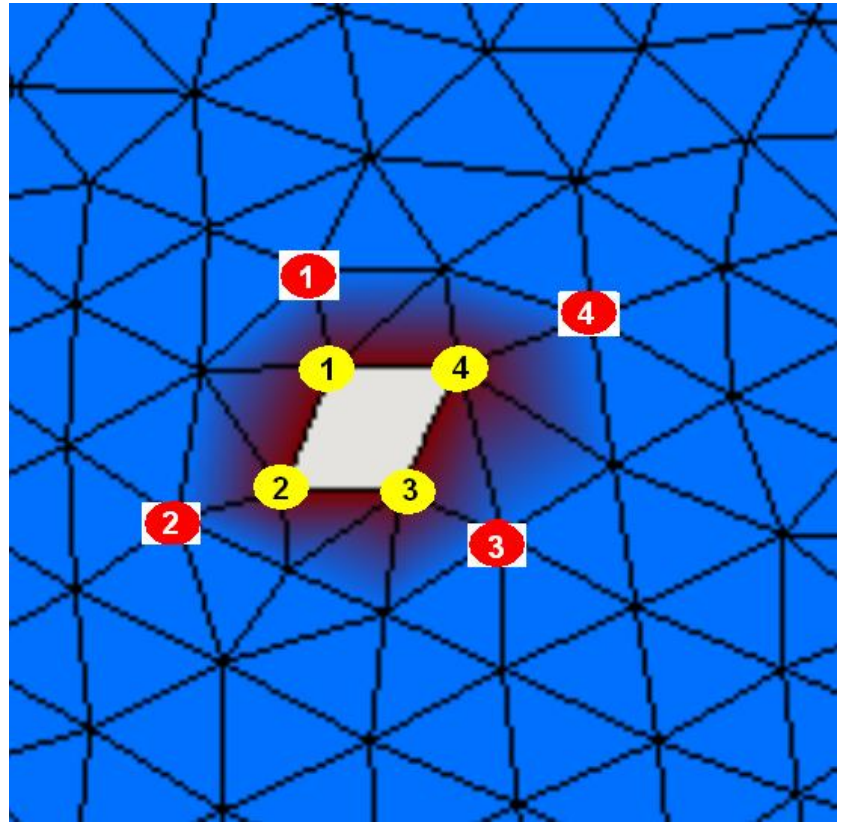

(a)

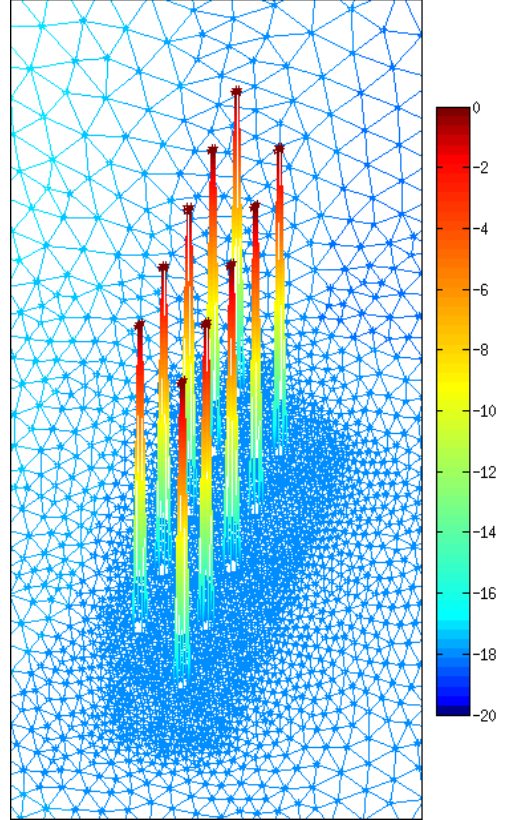

(b)

Figura 3: (a: Pontos para cálculo de velocidade, vermelhos e liberação de energia cinética, amarelos. (b) Forma dos conversores considerados pelo modelo, no caso o cenário 4

\subsection{Condições Iniciais e de Contorno}

O contorno oceânico é prescrito pelas marés astronômicas, elevação do nível da água do mar, velocidade da corrente, salinidade e temperatura. Os valores de salinidade e temperatura utilizados nas condições iniciais foram obtidos do Ocean Circulation and Climate Advanced Modeling Project (OCCAM).

A simulação foi iniciada com uma elevação de 0,5 metros que representa o nível médio da maré (K1, M2, N2, O1 e S2) para a região. A amplitude e fase para do contorno oceânico foram calculados com o Grenoble Model FES95.2 (Finite Element Solution v. 95.6).

Para descargas de rios, foram utilizados dados do Rio Guaíba, Rio Camaquã e o canal de São Gonçalo, com valores de vazão obtidos da Agência Nacional de Águas (ANA). A vazão do canal de São Gonçalo foi assumida como constante e igual a $760 \mathrm{~m}^{3} / \mathrm{s}$, devido à falta de dados [9].

No contorno superficial, as variações espaço-temporais do vento foram representadas através de dados da página do National Oceanic \& Atmospheric Administration (NOAA), interpolados e aplicados a cada nó do domínio do modelo numérico. De forma complementar, os dados de temperatura do ar junto à superfície do oceano foram obtidos da página da NOAA, e forçados de forma similar aos ventos, possibilitando que os processos de troca de calor com a atmosfera fossem levados em consideração no modelo.

\section{RESULTADOS E DISCUSSÃO}

De acordo com Kirinus [6], a região estudada apresenta uma grande convergência de diferentes massas de água, as quais tem uma velocidade média em torno de $0,4 \mathrm{~m} / \mathrm{s}$ e assim pode apresentar uma média de conversão de 10kW/dia ao longo de um ano.

Para se determinar a melhor geometria para o sítio de conversão foi analisado o campo de velocidade residual associado com a energia convertida no campo.

O padrão de circulação residual (Figura 4) é o mesmo em todas as simulações durante o período simulado, devido à predominância de vento sul. Atrás das turbinas pode ser observado uma zona de sombra, efeito denominado Wake ou esteira [10] formando vórtices que se deslocam com uma velocidade menor, podendo interferir em turbinas adjacentes diminuindo o potencial de energia total. 
Na Figura 4a, cenário 1, as turbinas estão posicionadas no sentido sudoeste-nordeste, estando praticamente alinhadas com a corrente, por conta disso a geração de energia elétrica é menor, sendo notada uma maior geração nas turbinas posicionadas ao sul e a sudeste do sítio. Possivelmente por conta do efeito esteira, as turbinas posicionadas a noroeste recebem velocidades de corrente incidente menores.

Na Figura 4b, cenário 2, as turbinas estão com uma pequena inclinação com relação a corrente residual, favorecendo a geração de energia elétrica, pois a interferência do efeito esteira na turbina adjacente é inferior.

No cenário 3, representado na Figura 4c, as turbinas estão praticamente ortogonais com relação a corrente, assim as turbinas posicionadas a sudoeste apresentam maior geração de energia elétrica com relação as turbinas posicionadas a nordeste, que sofrem o efeito Wake, reduzindo as taxas de conversão.

Apesar do cenário 4, Figura 4d, apresentar uma configuração diferente do cenário anterior, observamos médias de conversão parecidas. As turbinas estão na posição sul-norte, sendo que a corrente incide de forma quase paralela, apresentando maior geração nas turbinas ao sul e as posicionadas a leste. Novamente a zona de sombra formada atrás das turbinas a leste prejudica a velocidade da corrente incidente nas turbinas a oeste.

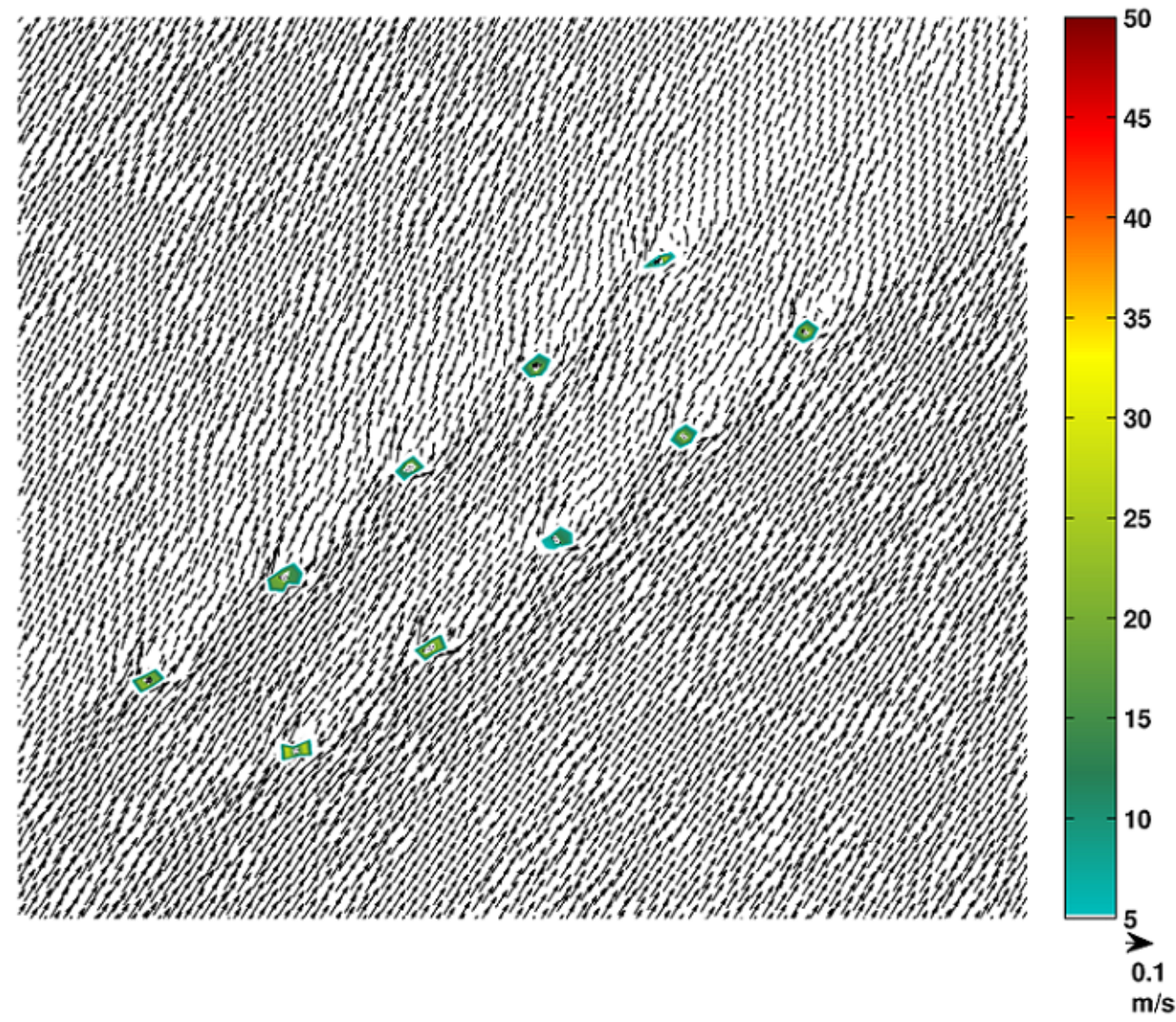

(a) 


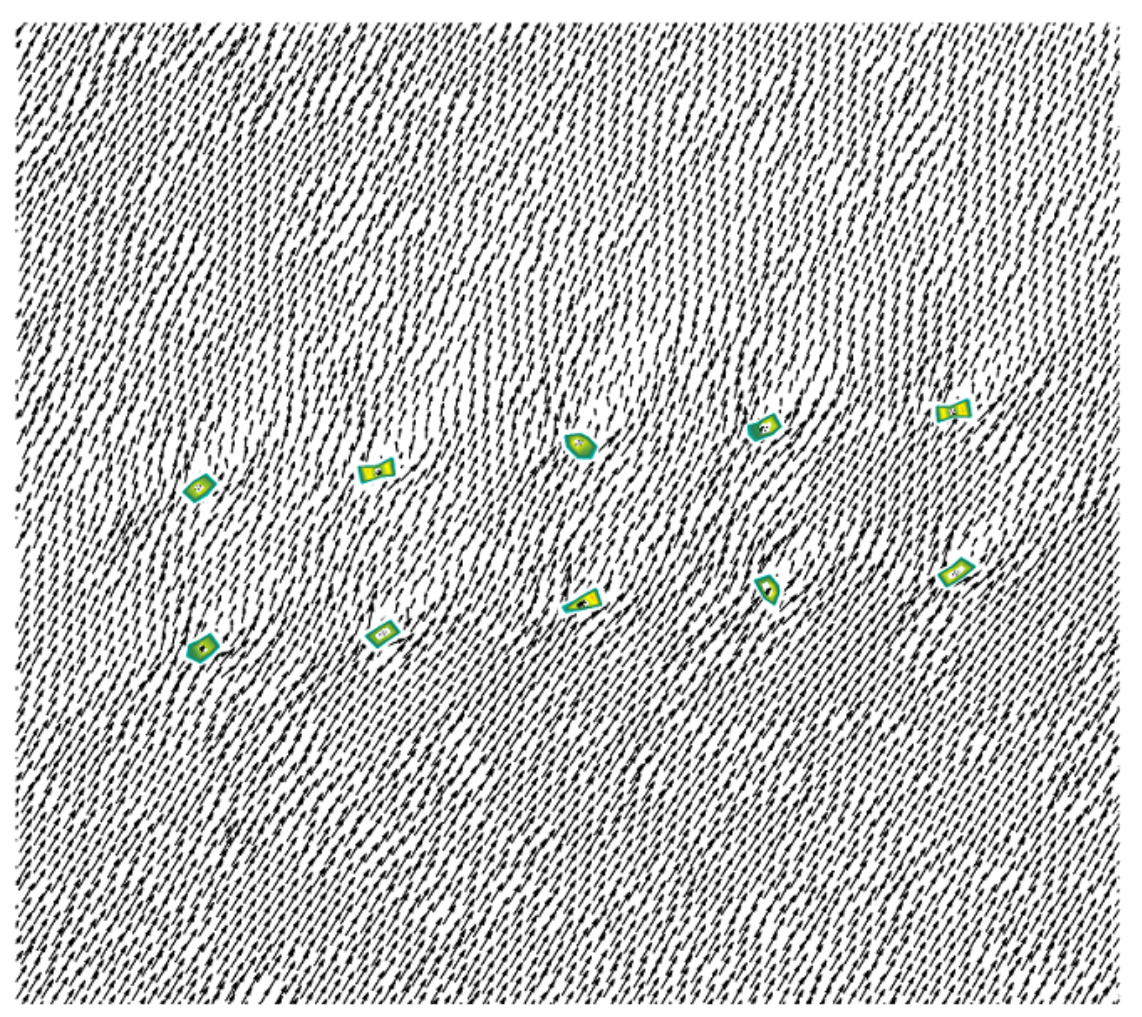

(b)

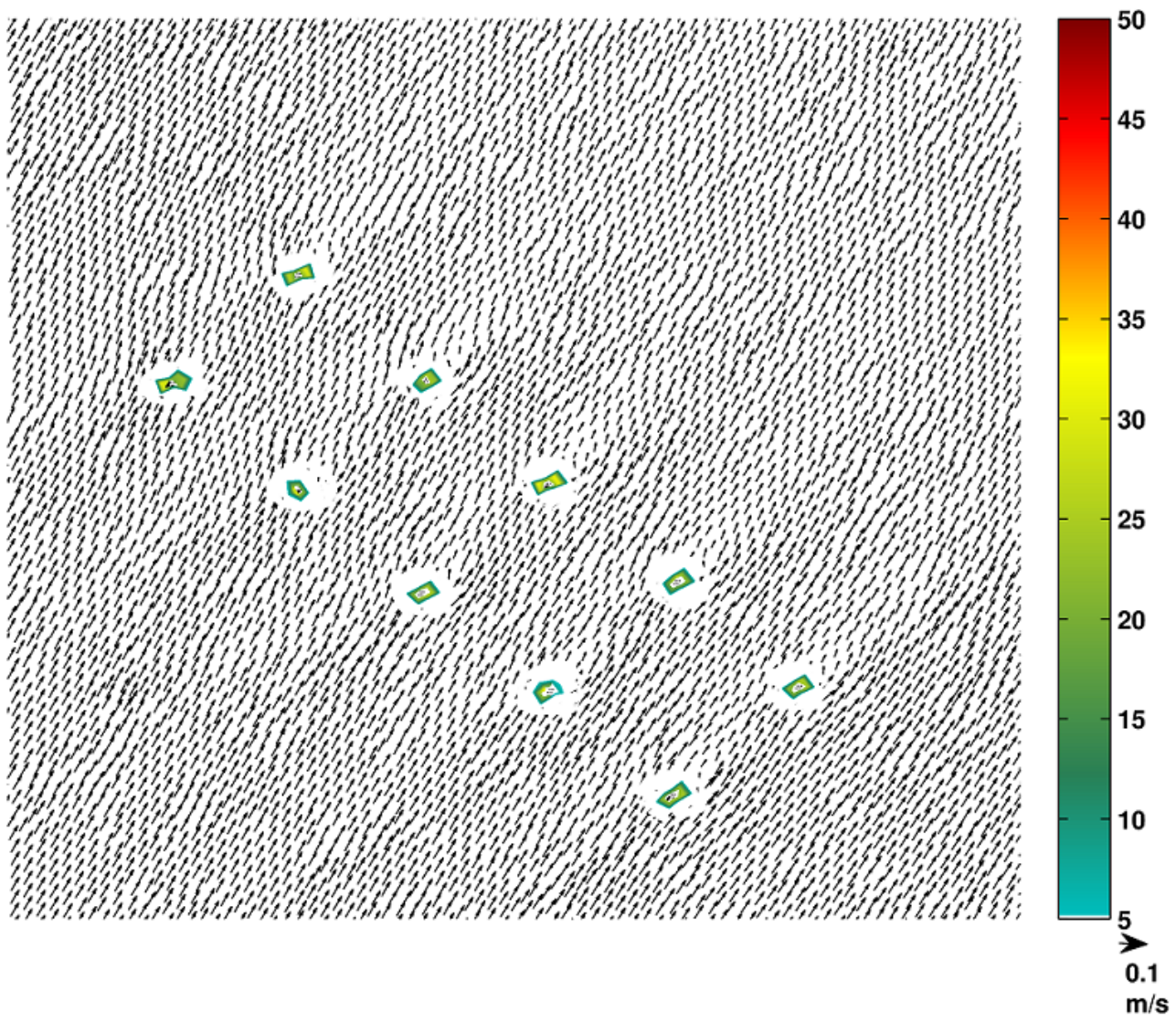

(c) 


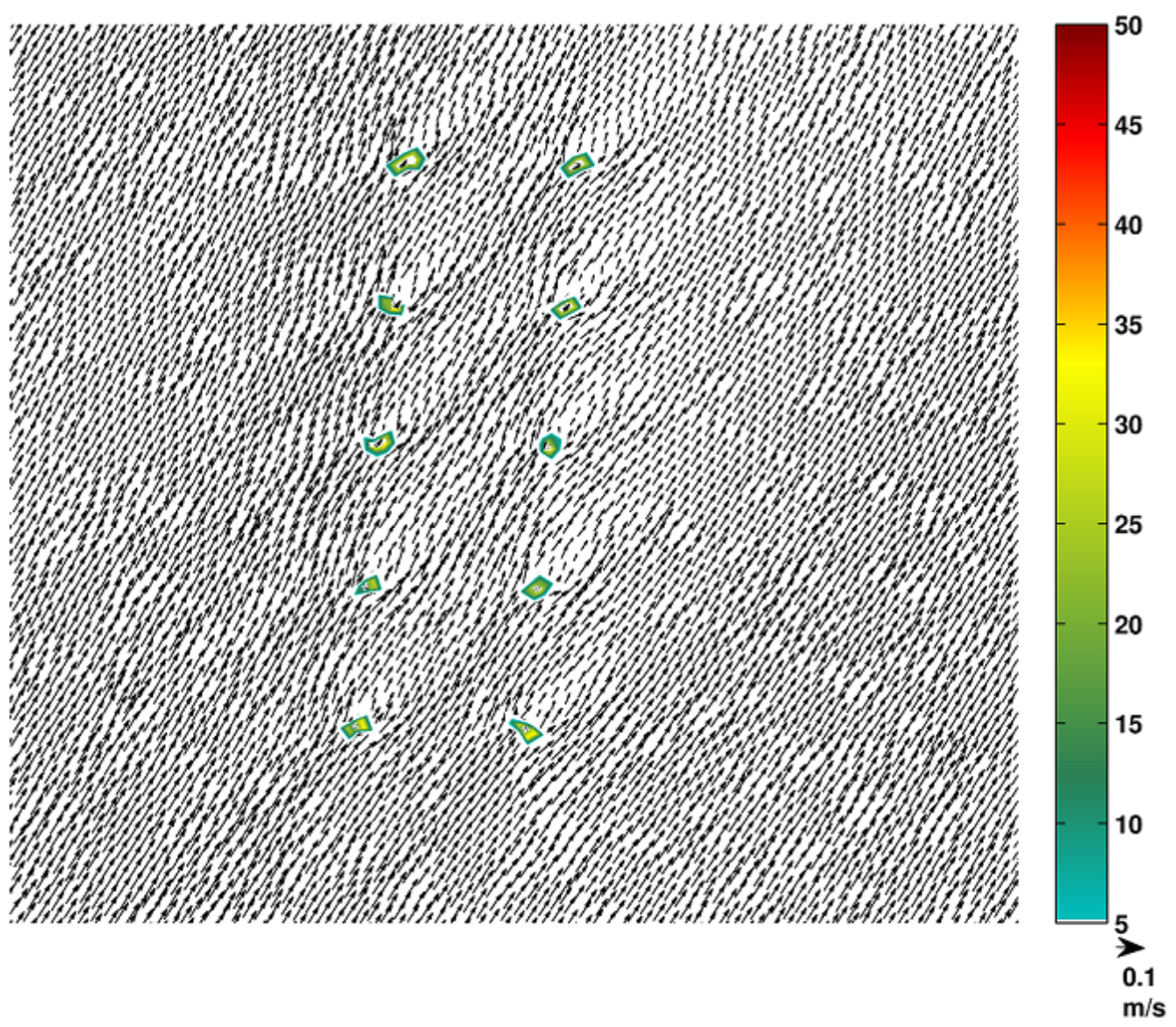

(d)

Figura 4: Direção e velocidade da corrente nos diferentes cenários. a) $0^{\circ}$ em relação à costa b) $45^{\circ}$ em relação à costa c) $90^{\circ}$ em relação à costa d) $135^{\circ}$ em relação à costa

Tabela 2: Valores de energia e frequência dos diferentes cenários

\begin{tabular}{cccccc}
\hline & Cenário & & & \\
\cline { 1 - 3 } Energia & $\boldsymbol{I}$ & II & & III & IV \\
\hline $\begin{array}{c}\text { Média }(k W) \\
\text { Desvio padrão } \\
(\mathrm{kW})\end{array}$ & 23,93 & 33,12 & 30,49 & 30,01 \\
\hline Integrado $(M W)$ & 14,36 & 44,12 & & 44,65 & 40,75 \\
\hline Frequência & $\boldsymbol{I}$ & 19,87 & 18,29 & 18,00 \\
\hline$<25 \mathrm{~kW}(\%)$ & 68,66 & $\boldsymbol{I I}$ & $\boldsymbol{I I I}$ & $\boldsymbol{I V}$ \\
\hline $25-40 \mathrm{~kW}(\%)$ & 13,33 & 11,16 & 10,33 & 13,17 \\
\hline$>40 \mathrm{~kW}(\%)$ & 18,00 & 29,33 & 24,66 & 25,67
\end{tabular}

De acordo com a Tabela 2, o cenário 1 apresentou menor rendimento, tendo uma média de $23,9 \mathrm{~kW}$ e um valor integrado de 14,6MW. Possivelmente devido a seu alinhamento com a corrente que acarretou em um predomínio de geração abaixo de $25 \mathrm{~kW}(68,6 \%)$. Este cenário foi o único a apresentar um desvio padrão abaixo de $40 \mathrm{~kW}$, fato que corrobora a afirmação anterior.

O cenário 2 demonstrou maior rendimento, tendo um valor integrado de 19,9MW de geração de energia elétrica. Provavelmente devido ao fato de que uma turbina interferiu pouco sobre as taxas de conversão de outra. Apresentando assim maior média de 33,1 kW. Seu desvio padrão foi menor em relação aos cenários 3 e 4, mas isso se deve ao fato de que suas frequências de geração foram mais constantes e apresentou a maior frequência de geração de energia elétrica 
acima de $40 \mathrm{~kW}(29,3 \%)$, demonstrando que este cenário possui alta estabilidade para conversão de energia no período estudado.

Os cenários 3 e 4, apesar de terem geometrias bem distintas apresentam valores de geração de energia elétrica próximos. Sendo que o cenário 3 apresenta uma pequena vantagem, possivelmente associada às turbinas posicionadas a leste, quase perpendiculares a direção da corrente.

\section{CONCLUSÃO}

Devido à grande dinâmica das correntes, influenciadas por ventos cíclicos (Nordeste/Sudeste), da PCSB, o uso de turbinas de geração de energia a partir de correntes oceânicas se torna viável. Neste trabalho concluímos que a melhor geometria para o sítio é a de $45^{\circ}$ em relação à costa. Entretanto simulações mais longas devem ser feitas para que se possa analisar a real influência dos ventos no decorrer de um ano sobre as correntes e consequentemente sobre o sítio de geração de energia.

\section{AGRADECIMENTOS}

Os autores do presente trabalho agradecem à Agência Nacional do Petróleo (ANP) e à Fundação de Amparo à Pesquisa do Estado do Rio Grande do Sul (FAPERGS) pelo financiamento parcial, através do pagamento de bolsas de estudo. Os autores agradecem também ao Conselho Nacional de Pesquisa $(\mathrm{CNPq})$ por dar suporte a este trabalho sob os contratos: 456292/2013-6 e 305885/2013-8.

\section{REFERÊNCIAS BIBLIOGRÁFICAS}

1. Bollmann M, Bosch T, Colijn F, Ebinghaus R, Froese R, Güssow K. World Ocean Review. Maribus, Hamburg, 1 edition. 2010.

2. Cruz JMBP, Sarmento AJNA. Sea State Characterization of the Test Site of an Offshore Wave Energy Plant. Ocean Engineering. 2007; (24):763-775.

3. Brown MA, Franchunk C, Koske BH, Michaels G, Pellegrino J. Results of a Technical Review of the U.S. Climate Change Technology Program's R\&D Portfolio. Technical report, Oak Ridge National Laboratory, Oak Ridge, Tennessee. 2006.

4. Capeletto GJ, De Moura GHZ. Balanço Energético do Rio Grande do Sul 2010: ano base 2009. 2010

5. Marques WC, Fernandes EHL, Malcherek A, Rocha LAO. Energy converting structures in the Southern Brazilian Shelf: Energy Conversion and its influence on the hydrodynamic and morphodynamic processes. Journal of Geophysical Research. 2012

6. Kirinus EP, Marques WC, Stringari CE.Viabilidade de conversão da energia de correntes marinhas na Plataforma Continental Sul do Brasil. 2012. Vetor, 22.

7. Piola AR, Romero SI, Zajaczkovski U. Space-time variability of the Plata plume inferred from ocean color. Continental Shelf Research. 2008; 28:1556-1567.

8. Hervouet JM. Free surface flows: Modelling with the finite element methods. England. 2007.

9. Viegas JS, Franz AFH. Hidrologia do Canal de São Gonçalo. 2006. Pelotas.

10. Ji B, Luo X, Wua Y, Peng X, Xu H. Partially-Averaged Navier-Stokes method with modified k-e model for cavitating flow around a marine propeller in anon-uniform wake. International Journal of Heat and Mass Transfer. 2012; 55:6582-6588. 